\title{
ON THE DIFFRACTION OF A SHOCK WAVE BY GENERAL TWO-DIMENSIONAL DISTURBANCES*
}

\author{
BY \\ Y. S. PAN \\ University of Tennessee Space Institute
}

\begin{abstract}
Introduction. Solutions for the diffraction of a plane shock wave by general twodimensional weak disturbances are presented. The technique employed here is the method developed by Ludloff and his associates for the aerodynamics of blasts [1]. They investigated the flow fields behind an advancing shock over stationary thin surfaces. The flow fields ahead of the shock are uniform and undisturbed; the flow fields behind the shock are disturbed only by the portion of the surfaces passed over by the shock. In this paper, the diffraction of a shock wave by weak disturbances caused by moving bodies are coinsidered. The solution shows that the flow fields behind the shock and the plane shock are disturbed not only by the portion of the body passed over by the shock but also by the portion of the body ahead of the shock. For a stationary body the present solution reduces to the corresponding solution in [1]. Thus the present solutions can be applied to many shock diffraction problems of practical interest, such as blast effects on aircrafts and on wings at angles of attack, moving subsonically or supersonically, diffraction of a shock due to nonsmooth moving walls in shock tubes, and diffraction of sonic booms due to nonplanar moving surfaces on the ground.
\end{abstract}

Weak disturbances are assumed to be described by the distributions of sources, doublets, and vortices in a two-dimensional problem, and by the distribution of point sources in an axisymmetric problem (see Fig. 1). Since the prescribed disturbances are wcak, the plane shock is only slightly disturbed, and the disturbed flow behind the shock is only slightly different from the undisturbed flow behind the shock. The disturbance pressure $p$ behind the shock wave is governed by a wave equation in three variables $(X, Y, T)$, where the coordinates are fixed with the undisturbed flow behind the shock. The shock conditions across the slightly disturbed shock yield a boundary condition $D_{X, T} p=G(Y, T)$ at $X=U T . D_{X, T}$ is a second-order linear hyperbolic differential operator of $X, T$ with constant coefficients and $G(Y, T)$ is a given function related to the prescribed disturbances ahead of the shock. By means of a Lorentz transformation of variables, $x, y, t$, the wave equation is preserved and the shock boundary condition reduces to $D_{x, t}^{*} p=G^{*}(y, t)$ at the shock $x=0$. The operator $D_{x, t}^{*}$ is of the same type as $D_{x, T}$.

* Received August 19, 1969; revised version received January 25, 1970. This work was carried out at New York University under the NASA Grant No. NGL-33-016-119. The author wishes to thank Dr. Ting for his suggestions, and also wishes to acknowledge the assistance of UTSI in the preparation of this paper. 


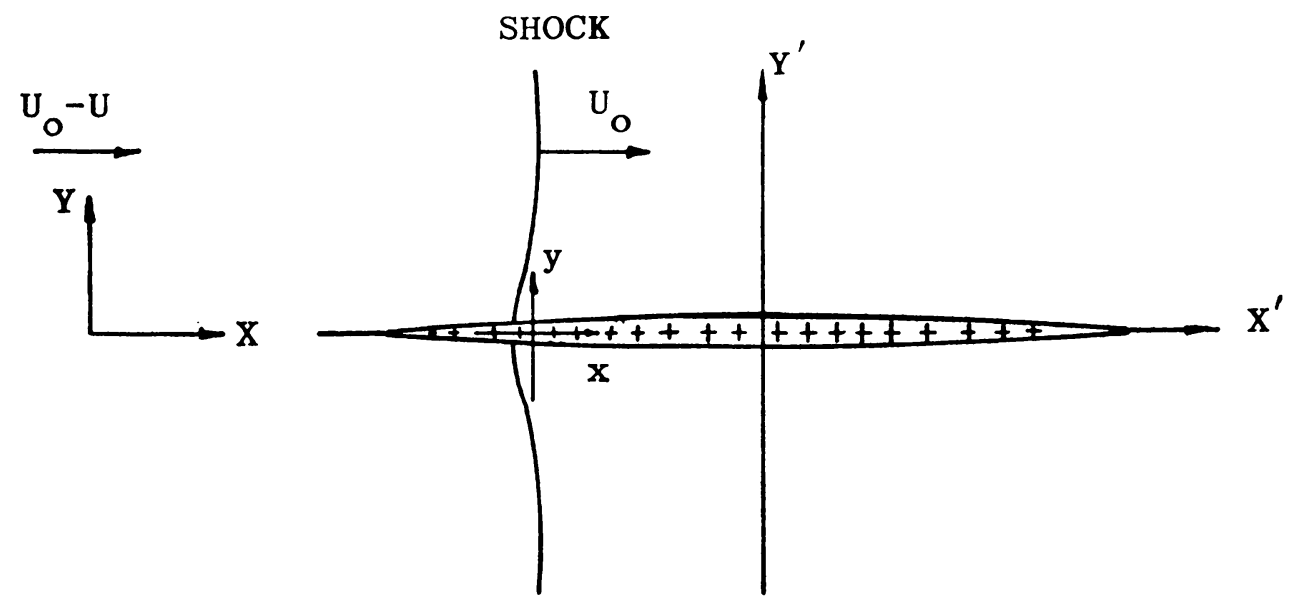

(a)

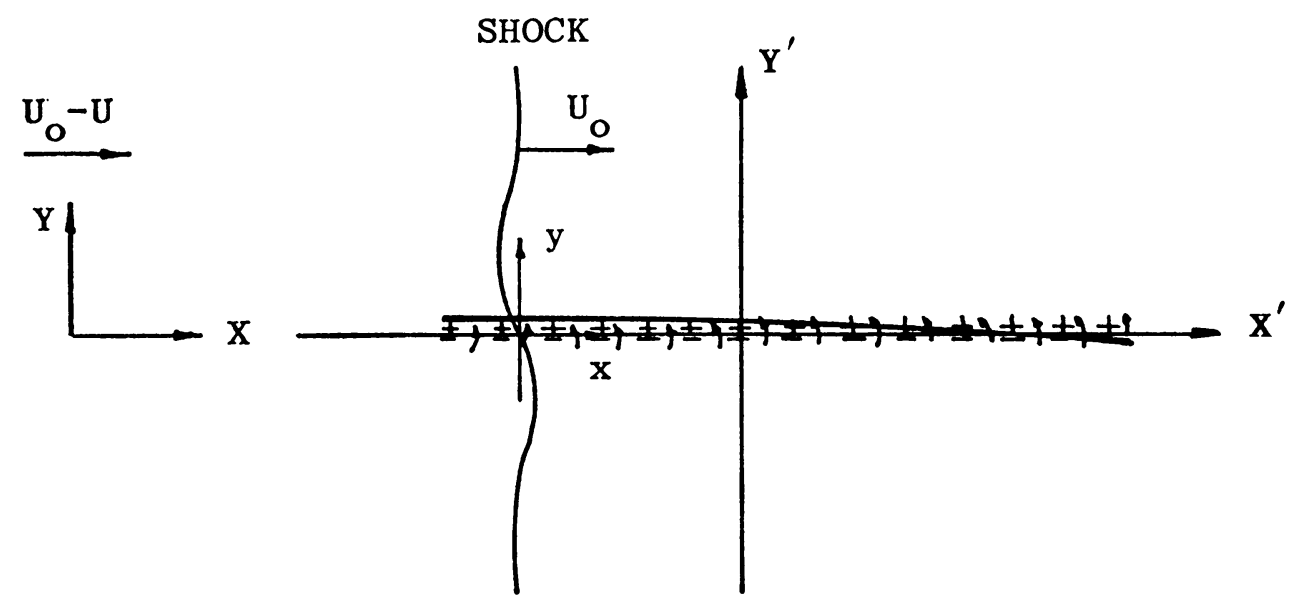

(b)

Fig. 1. Coordinate systems and prescribed disturbances

(a) Two-dimensional or axisymmetric source distribution

(b) Two-dimensional doublet or vortex distribution.

The prescribed general two-dimensional disturbances can be split into even, odd, and axisymmetric functions of $y$; accordingly, the disturbance pressure $p$ behind the shock can be divided in the same manner into even, odd, and axisymmetric solutions. They will be determined separately.

For the even solution of $p, p_{\nu}(x<0, y=0, t)$ has to be determined from the portion of the prescribed source distribution behind the shock (see Fig. 2). The disturbancs pressure, $p(x<0, y>0, t)$, as a solution of the wave equation can be expressed as an integral of the known distribution, $p_{y}\left(x<0, y=0^{+}, t\right)$, and an integral of the unknown distribution, $p_{v}\left(x>0, y=0^{+}, t\right)$, which is determined by the shock boundary condi- 


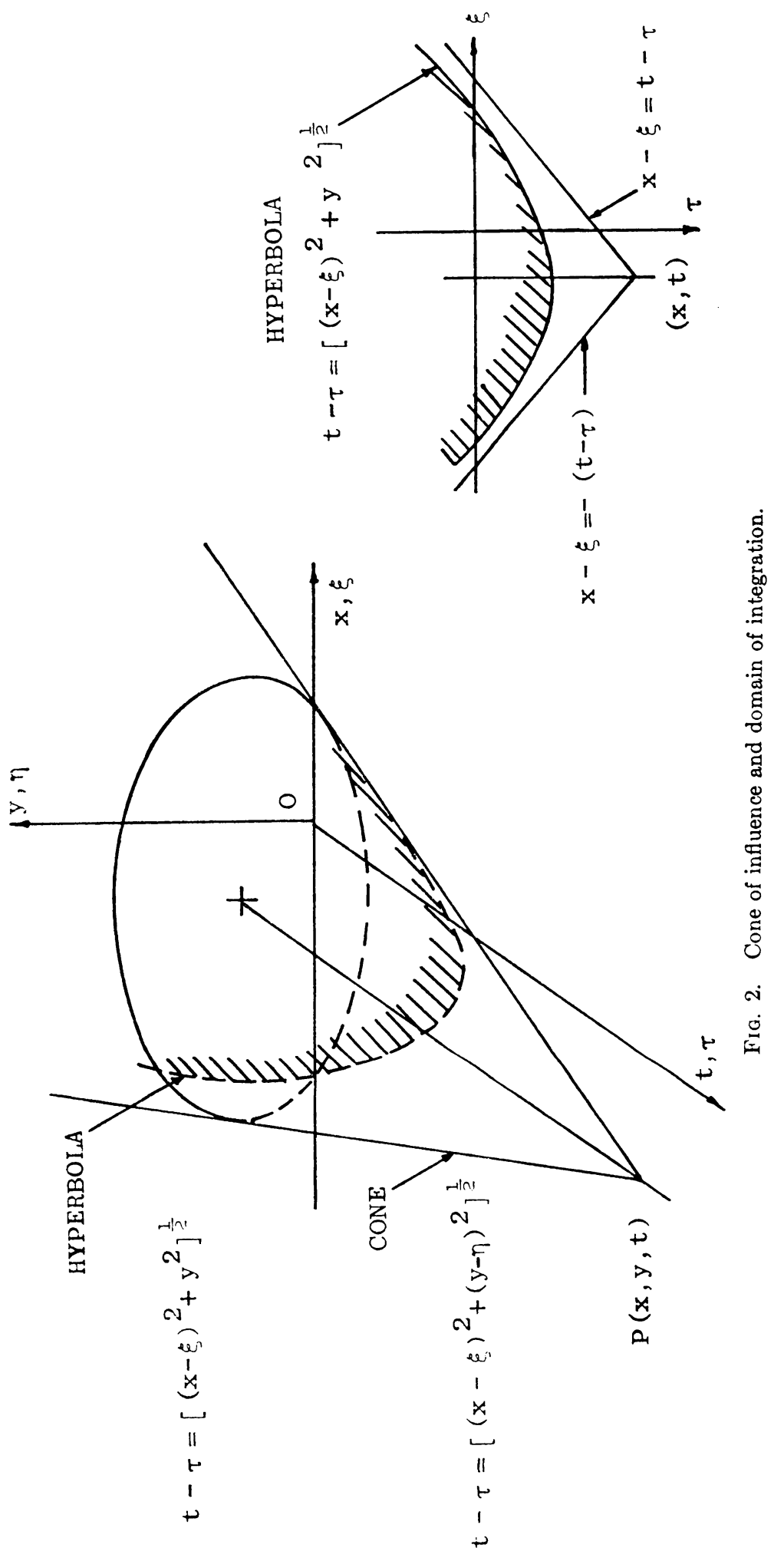


tion [1]. When the even disturbance is represented as an integral of the source distribution $f_{0}(X, T)$ on the plane $Y=0$, the shock boundary condition reduces to a differential equation $D_{x, t}^{*} p_{y}\left(x>0, y=0^{+}, t\right)=G^{*}\left(y=0^{+}, t\right)$, where $G^{*}\left(y=0^{+}, t\right)$ is a known function related to $f_{0}(X, T)$. Since the differential operator $D_{x, t}^{*}$ can be written as $\left((\partial / \partial x)-\lambda_{1}^{*}(\partial / \partial t)\right)\left((\partial / \partial x)-\lambda_{2}^{*}(\partial / \partial t)\right)$ with $\lambda_{1}^{*}, \lambda_{2}^{*}$ real, distinct and positive, solutions for $p_{y}\left(x>0, y=0^{+}, t\right)$ and hence for $p(x \leq 0, y, t)$ are obtained. For an odd or axisymmetric solution, the same technique is used with the source distribution replaced by a doublet (or a vortex) distribution or a point source distribution respectively.

The disturbance pressure behind the advancing shock is expressed by integrals of the prescribed disturbances. The shape of the diffracted shock and other disturbance quantities behind the shock are expressed in terms of the disturbance pressure behind the shock and the disturbances ahead of the shock. Applications to shock diffraction of thin structures in still air are shown.

1. Governing equations for disturbances behind the shock. We choose a moving coordinate system $(X, Y, T)$ fixed with the undisturbed flow behind the advancing shock wave (Fig. 1). The $X$-axis coincides with the shock propagation direction, and the $Y$-axis, being perpendicular to the $X$-axis, is the second coordinate of the general two-dimensional problem. The origin is so chosen that, at $T=0$, the shock hits the leading edge of the distributions of the prescribed disturbances. Then the flow behind the shock wave is governed by the usual general two-dimensional, unsteady Euler's equations.

As stated previously, the flow behind the advancing shock is only slightly disturbed due to the prescribed weak disturbances. We can linearize the Euler's equations to obtain the governing equations for disturbance pressure $p$, density $\rho$, and velocity components $u$ and $v$. They are

$$
\begin{aligned}
\frac{\partial \rho}{\partial T}+\frac{\partial}{\partial X}(R u) & +Y^{-i} \frac{\partial}{\partial Y}\left(R v Y^{i}\right)=0, \\
\frac{\partial u}{\partial T} & =-\frac{1}{R} \frac{\partial p}{\partial X} \\
\frac{\partial v}{\partial T} & =-\frac{1}{R} \frac{\partial p}{\partial Y} \\
\frac{\partial p}{\partial T} & =c^{2} \frac{\partial \rho}{\partial T} .
\end{aligned}
$$

Here $j=0$ for the two-dimensional case and $j=1$ for the axisymmetric case. $P, R$ and $c$ are respectively the pressure, density and speed of sound of the undisturbed flow behind the shock.

After elimination of $\rho, u$ and $v$ from the above equations (1.1), we have a wave equation for $p[1]$ :

$$
\square p=\left[\frac{\partial^{2}}{\partial T^{2}}+Y^{-i} \frac{\partial}{\partial Y}\left(Y^{i} \frac{\partial}{\partial Y}\right)-\frac{1}{c^{2}} \frac{\partial^{2}}{\partial T^{2}}\right] p=0 .
$$

$\rho, u$ and $v$ are satisfied by the following equations:

$$
\frac{\partial}{\partial T} \square \rho=\frac{\partial}{\partial T} \square u=\frac{\partial}{\partial T} \square v=0 .
$$


Now it is necessary to find two initial conditions and one boundary condition for $p$ to uniquely satisfy the wave equation (1.2) [1]. In the next section, we shall first prescribe the disturbances ahead of the shock. Boundary and initial conditions for the wave equation (1.2) will be given in Sec. III.

2. Prescribed disturbances. Ahead of the advancing shock wave, we choose a coordinate system $\left(X^{\prime}, Y^{\prime}\right)$ fixed with the undisturbed flow (Fig. 1). The $X^{\prime}$-axis coincides with the shock propagation direction (also $X$-axis) and the $Y^{\prime}$-axis is parallel to the $Y$-axis. The prescribed disturbances are generally weak in the sense that the disturbance velocities are much smaller than the speed of sound of the undisturbed flow ahead of the shock. We further assume that the disturbances can be expressed by a combination of distributions of sources, doublets, and vortices along the $X^{\prime}$-axis for a two-dimensional case and by a distribution of point sources along the $X^{\prime}$-axis for an axisymmetric case. The disturbances are irrotational and stationary with respect to $X^{\prime}, Y^{\prime}$; then the disturbance velocity potential and stream function exist and they satisfy the Laplace equation [2]. Since the problem is linear, we can treat the shock diffraction due to each distribution separately.

Suppose that the source distribution along the $X^{\prime}$-axis (in the plane $Y^{\prime}=0$ ) is specified by $f_{0}\left(X^{\prime}\right)$. It is well known that the velocity potential at any point $P\left(X^{\prime}, Y^{\prime}\right)$ can be written as

$$
\Phi_{s}\left(X^{\prime}, Y^{\prime}\right)=\frac{1}{4 \pi} \int_{-\infty}^{\infty} f_{0}\left(X_{1}^{\prime}\right) \ln \left[\left(X^{\prime}-X_{1}^{\prime}\right)^{2}+Y^{\prime 2}\right] d X_{1}^{\prime} .
$$

The above expression then may be written in the following "unsteady-like" form with a time coordinate $T^{\prime}$ :

$$
\Phi_{s}\left(X^{\prime}, Y^{\prime}\right)=-\frac{1}{2 \pi} \int_{-\infty}^{\infty} \int_{-\infty}^{T^{\prime \prime}-\left[\left(X^{\prime}-X_{1}^{\prime}\right)^{2}+Y^{\prime 2}\right\}^{1 / 2 / c}} \frac{c f_{0}\left(X_{1}^{\prime}\right) d T_{1}^{\prime} d X_{1}^{\prime}}{r_{1}^{\prime}}+\Phi_{0 .},
$$

with

$$
r_{1}^{\prime}=\left[c^{2}\left(T^{\prime}-T_{1}^{\prime}\right)^{2}-\left(X^{\prime}-X_{1}^{\prime}\right)^{2}-Y^{2}\right]^{1 / 2}
$$

and

$$
\Phi_{0 s}=\frac{1}{2 \pi} \int_{-\infty}^{\infty} f_{0}\left(X_{1}^{\prime}\right) \ln \left\{A+\left[A^{2}-\left(X^{\prime}-X_{1}^{\prime}\right)^{2}-Y^{\prime 2}\right]^{1 / 2}\right\}_{A \rightarrow \infty} .
$$

Here, $\Phi_{v s}$, which is due to the lower limit of the integration of $T_{1}^{\prime}$, is a number of very large magnitude; however, all orders of its differentiation with respect to its argument are zero. In the following analyses we shall be interested only in its differentiations; therefore we shall disregard this quantity.

The prescribed disturbances will be used to find a boundary condition at the shock in order to solve the disturbed flow behind the shock. The velocity potential is more appropriately expressed in the moving coordinates $(X, Y, T)$ fixed with the undisturbed flow behind the shock. The relations between the moving and stationary coordinates are

$$
X^{\prime}=X+\left(U_{0}-U\right) T, \quad Y^{\prime}=Y, \quad T^{\prime}=T,
$$

where $U_{0}$ and $U_{0}-U$ are respectively the velocities of the shock and the undisturbed flow behind the shock with respect to $X^{\prime}, Y^{\prime}$ coordinates. By changing the order of 
integrations, the velocity potential reduces to

$$
\Phi_{\triangleleft}(X, Y, T)=-\frac{1}{2 \pi} \int_{-\infty}^{T-Y / c} d T_{1} \int_{X-Z}^{X+Z} d X_{1} \frac{c f_{0}\left[X_{1}+\left(U_{0}-U\right) T\right]}{r_{1}}
$$

with $r_{1}=\left[z^{2}-\left(X-X_{1}\right)^{2}\right]^{1 / 2}$ and $Z=\left[c^{2}\left(T-T_{1}\right)^{2}-Y^{2}\right]^{1 / 2}$.

By using the same argument, we have the disturbance velocity potential or stream function due to doublet, vortex or point source distribution:

$$
\begin{aligned}
\Phi_{d}(X, Y, T) & =-\frac{1}{2 \pi} \frac{\partial}{\partial Y} \int_{-\infty}^{T-Y / c} d T_{1} \int_{X-Z}^{X+Z} d X_{1} \frac{c \mu_{0}\left[X_{1}+\left(U_{0}-U\right) T\right]}{r_{1}} . \\
\Psi(X, Y, T) & =\frac{1}{2 \pi} \int_{-\infty}^{T-Y / c} d T_{1} \int_{X-Z}^{X+Z} d X_{1} \frac{c \nu_{0}\left[X_{1}+\left(U_{0}-U\right) T\right]}{r_{1}}
\end{aligned}
$$

and

$$
\Phi_{a}(X, Y, T)=-\frac{1}{4 \pi} \int_{-\infty}^{\infty} d X_{1} \frac{g_{0}\left[X_{1}+\left(U_{0}-U\right) T\right]}{\left[\left(X-X_{1}\right)^{2}+Y^{2}\right]^{1 / 2}}
$$

Now it is clear that Eqs. (2.4) - (2.7) represent the velocity potential or the stream function at a point $(X, Y, T)$ due to a moving source $\left(f_{0}\right)$, doublet $\left(\mu_{0}\right)$, vortex $\left(\nu_{0}\right)$, or point source $\left(g_{0}\right)$ distribution.

The disturbance velocity components ahead of the shock are related to the disturbance velocity potential and stream function by

$$
\begin{aligned}
& u_{0}(X, Y, T)=\partial \Phi / \partial X=\partial \Psi / \partial Y, \\
& v_{0}(X, Y, T)=\partial \Phi / \partial Y=-\partial \Psi / \partial X .
\end{aligned}
$$

The disturbance pressure and density ahead of the shock can be found from the linearized Bernoulli's equation [2]. Since they are of the order of the square of the disturbance velocity, they are neglected in the analysis.

When the shock wave passes over the distributions which represent the disturbances due to a moving solid body, the strengths of these distributions are changed in the $(X, Y, T)$ coordinates. The strengths of the distributions passed over by the shock are related to the shape of the body and the undisturbed flow velocity relative to the body behind the shock. The expressions of the velocity potential and the stream function behind the shock are similar to Eqs. (2.4) to (2.7), provided that $f_{0}, \mu_{0}, \nu_{0}$ and $g_{0}$ are replaced by $f, \mu, \nu$ and $g$ respectively, and the lower limit of the integrals is replaced by a constant $(T=0)$.

\section{Boundary and initial conditions. ${ }^{1}$}

3.1. Boundary condition at shock. The shock boundary condition is carried out in the same manner as in [1]. Relative to the shock the prescribed disturbances are moving with constant velocity $-\left(U_{0}-U\right)$, while the undisturbed shock front can be expressed by

$$
X=U T+s(Y, T)
$$

Since the disturbances are weak, $s$ is a higher-order quantity in comparison with the

${ }^{3}$ For detailed analyses, readers are referred to [3]. 
first-order quantity $U T$. The shock angle is given by

$$
\theta=-\partial X / \partial Y=-s_{Y}(Y, T),
$$

a higher-order quantity. The shock velocity components are

$$
\begin{gathered}
U_{.}=d X / d T=U+S_{T}(Y, T), \\
v_{\mathbf{\bullet}}=U_{\mathbf{\bullet}} \tan \theta \cong U \theta .
\end{gathered}
$$

By using the oblique shock relations we find that the undisturbed quantities satisfy the usual normal shock relations and the disturbance quantities are related by the following linear equations:

$$
\begin{aligned}
c^{2} \rho & =\left(1+\Omega_{0}\right) p, \\
u & =\frac{\Omega_{1}}{R c} p+u_{0}, \\
-\left(U_{0}-U\right) s_{T} & =\frac{\Omega_{2}}{R} p+\frac{2 c}{\gamma+1} \frac{M^{2}-1}{M} u_{0}, \\
-\left(U_{0}-U\right) s_{Y} & =v-v_{0},
\end{aligned}
$$

where $R$ is the undisturbed flow density behind the shock,

$$
\Omega_{0}=-\frac{(\gamma-1)\left(M^{2}-1\right)^{2}}{M^{2}\left[(\gamma-1) M^{2}+2\right]}, \quad \Omega_{1}=\frac{(3 \gamma-1) M^{2}+(3-\gamma)}{2 M\left[(\gamma-1) M^{2}+2\right]} \quad \text { and } \quad \Omega_{2}=\frac{M^{2}-1}{2 M^{2}} \text {. }
$$

$u, v, \rho$ and $s$ in Eqs. (3.5) can be eliminated by linear acoustic equations; a boundary condition for $p$ alone to be applied at the shock $X=U T$ can be formulated:

$$
D_{X, T} p(X=U T, Y, T)=G(X=U T, Y, T),
$$

with

$$
\begin{aligned}
D_{X, T}=\left(\Omega_{1}+M+\Omega_{2} M\right) \frac{\partial^{2}}{\partial T^{2}}+\left(1+M^{2}+2 M\right. & \left.\Omega_{1}\right) c \frac{\partial^{2}}{\partial X \partial T} \\
& +M\left(1+\Omega_{1} M-\Omega_{2}\right) c^{2} \frac{\partial^{2}}{\partial X^{2}}
\end{aligned}
$$

and

$$
G(X=U T, Y, T)=-R c\left[\frac{\partial^{2} u_{0}}{\partial T^{2}}+M c \frac{\partial^{2} u_{0}}{\partial X \partial T}+\frac{2\left(1-M^{2}\right)}{\gamma+1} c^{2} \frac{\partial^{2} u_{0}}{\partial T^{2}}\right] .
$$

3.2. Boundary condition on the $X$-axis. Since prescribed disturbances are split as even, odd, and axisymmetric disturbances, the disturbances behind the shock can be considered as even, odd and axisymmetric respectively. In the two-dimensional problem, we can solve the wave equation in the region $-\infty<X<U T$ and $0 \leq Y<\infty$. Furthermore, the prescribed disturbances, being stationary with respect to $X^{\prime}, Y^{\prime}$, may be overtaken by the advancing shock. Therefore, a boundary condition on the $X$-axis behind the shock should be specified.

As stated previously, the disturbances considered are due to the motion of a solid body. The strengths of the distributions are changing while their velocities are unchanged 
across the shock. The velocity potential at any point behind the shock due to the source distribution $f(X, T)$ is

$$
\Phi_{\wedge}(X \leq U T, Y, T)=-\frac{1}{2 \pi} \int_{0}^{T-Y / c} d T_{1} \int_{X-Z}^{X+Z} d X_{1} \frac{c f\left[X_{1}+\left(U_{0}-U\right) T\right]}{r_{1}},
$$

where $T=0$ is the instant that the shock hits the leading edge of the source distribution, and

$$
f\left[X+\left(U_{0}-U\right) T\right]=0 \text { for } X>U T,
$$

i.e. the velocity potential at $(X \leq U T, Y, T)$ is affected only by the distribution behind the shock. As $Y \rightarrow 0, \Phi$, can be found as [4]:

$$
\Phi .(X \leq U T, Y \rightarrow 0, T)=\frac{1}{2}(c T-Y) f\left[X+\left(U_{0}-U\right) T\right]
$$

and the disturbance velocity $v$ is

$$
v(X \leq U T, Y \rightarrow 0, T)=\frac{1}{2} f\left[X+\left(U_{0}-U\right) T\right] .
$$

The boundary condition for $p$ on the $X$-axis is then

$$
p_{Y}(X \leq U T, 0, T)=-\frac{R c}{2} \frac{U_{0}-U}{c} f^{\prime}\left[X+\left(U_{0}-U\right) T\right],
$$

where the prime denotes the differentiation of a function with respect to its argument.

Similarly, the boundary condition on the $X$-axis behind the shock can be obtained for a doublet distribution:

$$
p(X \leq U T, 0, T)=-\frac{R c}{2} \frac{U_{0}-U}{c} \mu^{\prime}\left[X-\left(U_{0}-U\right) T\right]
$$

for a vortex distribution:

$$
p(X \leq I T, 0, T)=\frac{R c}{2} \frac{U_{0}-U}{c} \nu\left[X-\left(U_{0}-U\right) T\right]
$$

and for a point source distribution in axisymmetric case:

$$
Y p_{Y}(X \leq L T, 0, T)=-\frac{R c}{2 \pi} \frac{U_{0}-U}{c} g^{\prime}\left[X+\left(U_{0}-U\right) T\right] .
$$

3.3. Boundary condition at infinity.

$$
p(X \rightarrow-\infty, Y \rightarrow \infty, T)=0 .
$$

3.4. Initial conditions.

$$
\begin{gathered}
p(X \leq U T, Y, T \rightarrow-\infty)=0 \\
p_{T}(X \leq U T, Y, T \rightarrow-\infty)=0 .
\end{gathered}
$$

\section{Solution for disturbance pressure.}

4.1. The Lorentz transformation. We may introduce a new coordinate system $(x, y, t)$, such that the plane $x=0$ corresponds to the plane shock $X=U T$. The new coordinates are related to the old coordinates $(X, Y, T)$ by the Lorentz transformation 


$$
x=(X-U T)(1-M)^{-1 / 2}, \quad y=Y, \quad t=(c T-M X)\left(1-M^{2}\right)^{-1 / 2} .
$$

The wave equation (2.1) remains unchanged in $(x, y, t)$ :

$$
\left[\frac{\partial^{2}}{\partial t^{2}}-\frac{\partial^{2}}{\partial x^{2}}-y^{-i} \frac{\partial}{\partial y}\left(y^{i} \frac{\partial}{\partial y}\right)\right] p=0 .
$$

The boundary conditions become:

$$
\begin{gathered}
p \rightarrow 0 \text { as }\left(x^{2}+y^{\dot{2}}\right)^{1 / 2} \rightarrow \infty, \\
D_{x, t}^{*} p(x=0, y, t)=G^{*}(x=0, y, t) .
\end{gathered}
$$

Here $D_{x, t}^{*}$ is a linear differential operator of hyperbolic type,

$$
D_{x, t}^{*}=\frac{1}{M_{0}^{2}} \frac{\partial^{2}}{\partial t^{2}}+2 M \frac{\partial^{2}}{\partial x \partial t}+\frac{\partial^{2}}{\partial x^{2}},
$$

with $M_{0}^{2}=\left[2 \gamma M^{2}-(\gamma-1)\right] /\left[(\gamma-1) M^{2}+2\right]$ the Mach number of undisturbed flow ahead of the shock. $G^{*}(x=0, y, t)$ is related to the prescribed disturbances by (3.8). For an even disturbance (source distribution)

$$
G^{*}(x=0, y, t)=\frac{R c}{\pi}\left[\frac{4\left(\gamma M^{2}+1\right)}{(\gamma+1)^{2} M}\right] \int_{-\infty}^{t-y} d \tau \int_{-z}^{z} d \xi \frac{f_{0}^{\prime}(\zeta)_{x=0}}{\left(r_{1}^{*}\right)_{x-0}},
$$

where

$$
\zeta=\left[M \tau+\xi+M \frac{U_{0}-U}{U} t+M^{2} \frac{U_{0}-U}{U} x\right] /\left(1-M^{2}\right)^{1 / 2}
$$

and

$$
r_{1}^{*}=\left[z^{2}-(x-\xi)^{2}\right]^{1 / 2}=\left[(t-\tau)^{2}-y^{2}-(x-\xi)^{2}\right]^{1 / 2} .
$$

For an odd disturbance (doublet or vortex distribution)

$$
G^{*}(x=0, y, t)=-\frac{R c}{\pi}\left[\frac{4\left(\gamma M^{2}+1\right)}{(\gamma+1)^{2} M}\right] \frac{\partial}{\partial y} \int_{-\infty}^{t-\nu} d \tau \int_{-s}^{z} d \xi \frac{F_{0}^{\prime \prime}(\zeta)_{x-0}}{\left(r_{1}^{*}\right)_{x=0}}
$$

where $F_{0}(\zeta)=-\mu_{0}^{\prime}(\zeta)$ for doublet distribution, and $F_{0}(\zeta)=\nu_{0}(\zeta)$ for vortex distribution. For an axisymmetric disturbance (point source distribution)

$$
G^{*}(x=0, y, t)=-\frac{R c}{\pi}\left[\frac{4\left(\gamma M M^{2}+1\right)}{(\gamma+1)^{2} M\left(1-M^{2}\right)^{1 / 2}}\right] \int_{-\infty}^{\infty} d \xi \frac{g_{0}^{\prime \prime \prime}(\zeta)_{x-0}}{\left[\xi^{2}+y^{2}\right]^{1 / 2}} .
$$

The boundary conditions on $x$-axis for an even disturbance from Eq. (3.12)

$$
p_{\nu}(x \leq 0,0, t)=-\frac{R c}{2} \frac{U_{0}-U}{c} f^{\prime}\left[a_{0}^{*}\left(\lambda_{0}^{*} x+t\right)\right],
$$

where

$$
a_{0}^{*}=U_{0} / c\left(1-M^{2}\right)^{1 / 2} ; \quad \lambda_{0}^{*}=\left(1-M^{2}+\frac{M U_{0}^{*}}{c}\right) \frac{c}{U_{0}} .
$$

For an odd disturbance, from Eqs. (3.13) and (3.14), 


$$
p(x \leq 0,0, t)=\frac{R c}{2} \frac{U_{0}-U}{c} F\left[a_{0}^{*}\left(\lambda_{0}^{*} x+t\right)\right] .
$$

For an axisymmetric disturbance, from Eq. (3.15),

$$
y p_{\nu}(x \leq 0,0, t)=-\frac{R c}{2 \pi} \frac{U_{0}-U}{c} g^{\prime}\left[a_{0}^{*}\left(\lambda_{0}^{*} x+t\right)\right] .
$$

The two initial conditions Eqs. (3.1) and (3.18) are now

$$
p=p_{\iota}=0 \text { for } t \rightarrow-\infty .
$$

4.2. The Possio integral-two-dimensional problem. In general, the solution of such two-dimensional boundary initial value problems as the one defined by Eqs. (4.2), (4.3), (4.4), (4.14) and (4.10) (or (4.12)) can be solved in terms of "temporary sources (or doublet)" spread over a certain area in the $x-t$ plane characterizing the motion of the disturbances. Such solutions can be written as Possio integrals [1]. For the even solution,

$$
\begin{aligned}
p(x \leq 0, y>0, t)= & -\frac{1}{\pi} \int_{-\infty}^{t-\nu} d \tau \int_{x-s}^{0} d \xi \frac{p_{\nu}(\xi \leq 0,0, \tau>0)}{r_{1}^{*}} \\
& -\frac{1}{\pi} \int_{-\infty}^{t-\left(x^{2}+\nu^{2}\right)^{1 / 2}} d \tau \int_{0}^{x+z} d \xi \frac{p_{\nu}(\xi>0,0, \tau)}{r_{1}^{*}} .
\end{aligned}
$$

For the odd solution,

$$
\begin{aligned}
p(x \leq 0, y>0, t)= & -\frac{1}{\pi} \frac{\partial}{\partial y} \int_{-\infty}^{t-\nu} d \tau \int_{x-z}^{0} d \xi \frac{p(\xi \leq 0,0, \tau>0)}{r_{1}^{*}} \\
& -\frac{1}{\pi} \frac{\partial}{\partial y} \int_{-\infty}^{t-\left(x^{2}+y^{2}\right)^{2 / s}} d \tau \int_{0}^{x+z} d \xi \frac{p(\xi>0,0, \tau)}{r_{1}^{*}} .
\end{aligned}
$$

The integration area in the $\xi, \tau$ plane is confined by the hyperbola (see Fig. 2) $t-\tau=$ $\left[(x-\xi)^{2}-y^{2}\right]^{1 / 2}$ and the straight line $\tau=-\infty$. Here, $p_{y}$ or $p$ is given for the left half of the plane $y=0(x \leq 0)$ while it is unknown in the right half of the plane $(x>0)$. The next step is to find an equation for $p_{\nu}(x>0,0, t)$ or $p(x>0,0, t)$ which will replace shock boundary condition Eq. (4.5) prescribed at the plane $x=0$.

For even disturbances, by substituting Eq. (4.10) into Eq. (4.15), we have

$$
\begin{aligned}
p(x \leq 0, y>0, t)=-\frac{1}{\pi} \int_{-\infty}^{t-\left(x^{2}+\nu^{2}\right)^{2 / z}} d \tau \int_{0}^{x+s} d \xi \frac{p_{\nu}(\xi>0,0, \tau)}{r_{1}^{*}} & \\
& +\frac{R c}{2 \pi} \frac{U_{0}-U}{c} \int_{-\infty}^{t-\nu} d \tau \int_{x-z}^{0} d \xi \frac{f^{\prime}\left[a_{0}^{*}\left(\lambda_{0}^{*} \xi+\tau\right)\right]}{r_{1}^{*}} .
\end{aligned}
$$

To find an equation for $p_{\nu}(\xi>0,0, \tau)$, let us apply the shock boundary condition, Eq. (4.4):

$$
\begin{aligned}
-\int_{-\infty}^{t-\nu} d \tau \int_{\infty}^{z} d \xi \frac{D_{\xi .}^{*} p_{y}(\xi>0,0, \tau)}{r_{1}^{*}} & \\
+ & \frac{R c}{2} \frac{U_{0}-U}{c} a_{0}^{* 2} H\left(-\lambda_{0}^{*}\right) \int_{-\infty}^{t-\nu} d \tau \int_{0}^{z} d \xi \frac{f^{\prime \prime \prime}\left[a_{0}^{*}\left(\tau-\lambda_{0}^{*} \xi\right)\right]}{\left(r_{1}^{*}\right)_{x-0}} \\
- & \int_{-\infty}^{t-\nu} d \tau \frac{\left[p_{\nu \xi}\left(0^{+}, 0, \tau\right)+2 M p_{\nu \tau}\left(0^{+}, 0, \tau\right)+\frac{R c}{2} \frac{U_{0}-U}{c} a_{0}^{*}\left(\lambda_{0}^{*}+2 M\right) f^{\prime \prime}\left(a_{0}^{*} t\right)\right]}{\left(r_{1}^{*}\right)_{x-\xi}}
\end{aligned}
$$




$$
=R c\left[\frac{4\left(\gamma M^{2}+1\right)}{(\gamma+1)^{2} M}\right] \int_{-\infty}^{t-\nu} d \tau \int_{0}^{z} d \xi \frac{\left[f_{0}^{\prime \prime \prime}\left(\zeta_{\xi-\xi}\right)_{x=0}+f_{0}^{\prime \prime \prime}\left(\zeta_{\zeta--\xi}\right)_{x=0}\right]}{r_{1}^{*}},
$$

where $H\left(\lambda_{0}^{*}\right)=M_{0}^{-2}-2 M \lambda_{0}^{*}+\lambda_{0}^{* 2}$. This shock boundary condition can be satisfied provided that

$$
\begin{aligned}
D_{\xi, \tau} p_{\nu}(\xi> & 0,0, \tau)=\frac{R c}{2} \frac{U_{0}-U}{c} a_{0}^{*} H\left(-\lambda_{0}^{*}\right) f^{\prime \prime \prime}\left[a_{0}^{*}\left(\tau-\lambda_{0} \xi\right)\right] \\
& -R c \frac{4\left(\gamma M^{2}+1\right)}{(\gamma+1)^{2} M} f_{0}^{\prime \prime \prime}\left[a^{*}\left(\tau+\lambda^{*} \xi+k t\right)\right]+f_{0}^{\prime \prime \prime}\left[a^{*}\left(\tau-\lambda^{*} \xi+k t\right)\right]
\end{aligned}
$$

with $a_{0}^{*}=U_{0} / c(1-M)^{1 / 2}, a_{1}^{*}=U / c\left(1-M^{2}\right)^{1 / 2}, \lambda^{*}=1 / M$, and $k=\left(U_{0}-U\right) / U$; and

$$
p_{\nu \xi}\left(0^{+}, 0, \tau\right)+2 M I p_{y \tau}\left(0^{+}, 0, \tau\right)=-\frac{R c}{2} \frac{U_{0}-U}{c} a_{0}^{*}\left(\lambda_{0}^{*}+2 M\right) f^{\prime \prime}\left(a_{0}^{*} \tau\right) .
$$

Now the problem is reduced to obtaining the function $p_{\nu}(\xi>0,0, \tau)$ which satisfies Eq. (4.19) and two boundary conditions at $\xi$ or $x=0^{+}, y=0$. These two boundary conditions can be obtained by a kind of "mean value theorem" at $x=0$ [1] and by Eq. (4.20). They are

$$
p_{\nu}\left(0^{+}, 0, \tau\right)=R c\left[\left(\frac{2 Y^{2}}{M^{2}-1} \frac{U_{0}}{c}+\frac{U_{0}-U}{2 c}\right) f^{\prime}\left(a_{0}^{*} \tau\right)-\left(\frac{2 M I^{2}}{M^{2}-1} \frac{U_{0}}{c}+\frac{4 M}{\gamma+1}\right) f_{0}^{\prime}\left(a_{0}^{*} \tau\right)\right]
$$

and

$$
\begin{aligned}
p_{\nu \xi}\left(0^{+}, 0, \tau\right)=-R c\left\{\left[\frac{4 M^{3}}{M^{2}-1} \frac{U_{0}}{c}+\right.\right. & \left.\frac{U_{0}-U}{2 c}\left(\lambda_{0}^{*}+4 M\right)\right] a_{0}^{*} f^{\prime \prime}\left(a_{0}^{*} \tau\right) \\
& \left.-\left[\frac{4 M^{3}}{M^{2}-1} \frac{L_{0}}{c}+\frac{8 M^{2}}{\gamma+1}\right] a_{0}^{*} f^{\prime \prime}\left(a_{0}^{*} \tau\right)\right\} .
\end{aligned}
$$

It can be shown that the solution for Eq. (4.19) has the form

$$
\begin{array}{rl}
p_{\nu}(\xi>0, y=0, \tau)=R & R c\left\{\sum_{i=0,1,2} A_{i} f^{\prime}\left[a_{0}^{*}\left(\tau-\lambda_{i} \xi\right)\right]+\sum_{i=1,2} B_{i} f_{0}^{\prime}\left[a_{0}^{*}\left(\tau-\lambda_{i}^{*} \xi\right)\right]\right. \\
& \left.+\sum_{i=1,2} C_{i} f_{0}^{\prime}\left[a^{*}\left(\tau-\lambda_{i}^{*} \xi+k t\right)\right]\right\} \\
& -\operatorname{Rc} \frac{1}{a^{*^{2}}} \frac{4\left(\gamma M^{2}+1\right)}{(\gamma+1)^{2} M}\left\{\frac{1}{H\left(-\lambda^{*}\right)} f_{0}^{\prime}\left[a^{*}\left(\tau+\lambda^{*} \xi+k t\right)\right]\right. \\
& \left.+\frac{1}{H\left(\lambda^{*}\right)} f_{0}^{\prime}\left[a^{*}\left(\tau-\lambda^{*} \xi+k t\right)\right]\right\} .
\end{array}
$$

Here $\lambda_{1}^{*}$ and $\lambda_{2}^{*}$ are two real, distinct, and positive roots of the quadratic equation

$$
H(\lambda)=\lambda^{2}+2 M \lambda+M_{v}^{-2}=0 .
$$

$A_{0}=\left(\left(U_{0}-U\right) / 2 c\right)\left(H\left(-\lambda_{0}^{*}\right) / H\left(\lambda_{0}^{*}\right)\right), A_{1}$ and $A_{2}$ are the solution of the two simultaneous linear equations

$$
A_{1}+A_{2}=\frac{2 M^{2}}{M^{2}-1} \frac{U_{0}}{c}+\frac{U_{0}-U}{2 c}\left[1-\frac{H\left(-\lambda_{0}^{*}\right)}{H\left(\lambda_{0}^{*}\right)}\right]
$$




$$
\lambda_{1}^{*} A_{1}+\lambda_{2}^{*} A_{2}=\frac{4 M^{3}}{M^{2}} \frac{U_{0}}{c}+\frac{U_{0}-U}{2 c}\left\{4 M+\lambda_{0}^{*}\left[1-\frac{H\left(-\lambda_{0}^{*}\right)}{H\left(\lambda_{0}^{*}\right)}\right]\right\} .
$$

$B_{1}$ and $B_{2}$ are the solution of the other two simultaneous linear equations

$$
\begin{aligned}
B_{1}+B_{2} & =-\frac{2 M^{2}}{M^{2}-1} \frac{U_{0}}{c}-\frac{4 M}{\gamma+1}, \\
\lambda_{1}^{*} B_{1}+\lambda_{2}^{*} B_{2} & =-\frac{4 M^{3}}{M^{2}-1} \frac{U_{0}}{c}-\frac{8 M}{\gamma+1} .
\end{aligned}
$$

$C_{1}$ and $C_{2}$ are the solution of the other two simultaneous linear equations

$$
\begin{aligned}
C_{1}+C_{2} & =\frac{4\left(\gamma M^{2}+1\right)}{(\gamma+1)^{2} M} \frac{1}{\mathrm{a}^{*^{2}}}\left[\frac{1}{H\left(-\lambda^{*}\right)}+\frac{1}{H\left(\lambda^{*}\right)}\right], \\
\lambda_{1}^{*} C_{1}+\lambda_{2}^{*} C_{2} & =-\frac{4\left(\gamma M^{2}+1\right)}{(\gamma+1)^{2}} M \frac{\lambda^{*}}{a^{*^{2}}}\left[\frac{1}{H\left(-\lambda^{*}\right)}-\frac{1}{H\left(\lambda^{*}\right)}\right] .
\end{aligned}
$$

For odd disturbances, we can find $p(\xi>0,0, \tau)$ in Eq. (4.16) by using a similar technique.

$$
\begin{aligned}
p(\xi>0, y=0, \tau)=-R c \mid & \sum_{i=0,1,2} A_{i} F\left[a_{0}^{*}\left(\tau-\lambda_{i}^{*} \xi\right)\right]+\sum_{i=1,2} B_{i} F_{0}\left[a_{0}^{*}\left(\tau-\lambda_{i}^{*} \xi\right)\right] \\
+ & \left.\sum_{i=1,2} C_{i} F_{0}\left[a^{*}\left(\tau-\lambda_{i}^{*} \xi+k t\right)\right]\right\} \\
+ & R c \frac{1}{a^{*^{2}}} \frac{4\left(\gamma M^{2}+1\right)}{(\gamma+1)^{2} M}\left\{\frac{1}{H\left(-\lambda^{*}\right)} F_{0}\left[a^{*}\left(\tau+\lambda^{*} \xi+k t\right)\right]\right. \\
+ & \left.\frac{1}{H\left(\lambda^{*}\right)} F_{0}\left[a^{*}\left(\tau-\lambda^{*} \xi+k t\right)\right]\right\},
\end{aligned}
$$

where $A_{i}, B_{i}, C_{i}$ and $\lambda_{i}^{*}$ are defined in the case for even disturbances.

4.3 Retarded potential-axisymmetric problem. By Kirchhoff's theorem, a solution of axisymmetric wave equation can be written in terms of retarded potential [5]. By using the boundary condition Eq. (4.13) on the $x$-axis, we have

$$
\begin{aligned}
\left.p(x \leq 0, y, t)=-\frac{1}{2} \int_{0}^{\infty} d \xi \frac{y p_{\nu}(\xi}{}>0,0, t-r_{2}^{*}\right) & \\
r_{2}^{*} & +\frac{R c}{4 \pi} \frac{U_{0}-U}{c} \int_{-\infty}^{\infty} d \xi \frac{g^{\prime}\left[a_{0}^{*}\left(\lambda_{0}^{*} \xi+t-r_{2}^{*}\right)\right]}{r_{2}^{*}},
\end{aligned}
$$

where $r_{2}^{*}=\left[(x-\xi)^{2}+y^{2}\right]^{1 / 2}$. As stated previously, we can apply the shock boundary condition Eq. (4.4) to find $y p_{y}(\xi>0,0, t)$. The result is

$$
\begin{aligned}
& y p_{\nu}\left(\xi>0,0, t-r_{2}^{*}\right)=-\frac{R c}{\pi}\left\{\sum_{i=0,1,2} A_{i} g^{\prime}\left[a_{0}^{*}\left(t-\left(\xi^{2}+y^{2}\right)^{1 / 2}-\lambda_{i}^{*} \xi\right)\right]\right. \\
& \left.+\sum_{i=1,2} B_{i} g_{0}^{\prime}\left[a_{0}^{*}\left(t-\left(\xi^{2}+y^{2}\right)^{1 / 2}-\lambda_{i}^{*} \xi\right)\right]+\sum_{i=1,2} D_{i} g_{0}^{\prime}\left[a_{0}^{*}\left(t-\lambda_{i}^{*} \xi\right)\right]\right\} \\
& \quad-\frac{R c}{\pi} \frac{1}{a_{0}^{* 2}} \frac{4\left(\gamma M^{2}+1\right)}{\left(1-M^{2}\right)^{1 / 2}(\gamma+1)^{2} M}\left\{\frac{1}{H\left(-\lambda_{3}^{*}\right)} g_{0}^{\prime}\left[a_{0}^{*}\left(t+\lambda_{3}^{*} \xi\right)\right]+\frac{1}{H\left(\lambda_{3}^{*}\right)} g_{0}^{\prime}\left[a_{0}^{*}\left(t-\lambda_{3}^{*} \xi\right)\right]\right\},
\end{aligned}
$$


where $\lambda_{3}^{*}=c / U_{0}=\lambda^{*} U / U_{0} \cdot \lambda_{i}^{*}, A_{i}$ and $B_{i}$ are defined in the case for even disturbances. $D_{1}$ and $D_{2}$ are the solution of the following two simultaneous linear equations:

$$
\begin{aligned}
D_{1}+D_{2} & =\frac{1}{a_{0}^{* 2}} \frac{4\left(\gamma M^{2}+1\right)}{\left(1-M^{2}\right)^{1 / 2}(\gamma+1)^{2} M}\left[\frac{1}{H\left(-\lambda_{3}^{*}\right)}+\frac{1}{H\left(\lambda_{3}^{*}\right)}\right], \\
\lambda_{1}^{*} D_{1}+\lambda_{2}^{*} D_{2} & =-\frac{\lambda_{3}^{*}}{a_{0}^{* 2}} \frac{4\left(\gamma M^{2}+1\right)}{\left(1-M^{2}\right)^{1 / 2}(\gamma+1)^{2} M}\left[\frac{1}{H\left(-\lambda_{3}^{*}\right)}-\frac{1}{H\left(\lambda_{3}^{*}\right)}\right] .
\end{aligned}
$$

5. Final results. The results of $p(x, y, t)$ for even, odd and axisymmetric disturbances can be obtained by substituting Eqs. (4.23), (4.28) and (4.30) into Eqs. (4.16), (4.17) and (4.29) respectively. By using the Lorentz transformation, Eq. (4.1), the disturbance pressures $p(X, Y, T)$ can be obtained from $p(x, y, t)$. From the linear relations (1.1) and the shock relations (3.5), the following disturbance quantities are obtained: the disturbance density

$$
c^{2} \rho(X, Y, T)=p(X, Y, T)+\Omega_{0} p(X, Y, T=X / U),
$$

the form of shock front

$$
\begin{array}{rl}
X=U T-\frac{\Omega_{2}}{R\left(U_{0}-U\right)} \int_{-\infty}^{T} & p(X=U \tau, Y, \tau) d \tau \\
& \quad-\frac{2 c}{U_{0}-U} \frac{M^{2}-1}{(\gamma+1)} \frac{1}{M} \int_{-\infty}^{T} u_{0}(X=U \tau, Y, T) d \tau,
\end{array}
$$

and the disturbance velocity components,

$u(X, Y, T)=-\frac{1}{R} \int_{X / U}^{T} p_{Y}(X, Y, \tau) d \tau+\frac{\Omega_{1}}{R c} p\left(X, Y, T=\frac{X}{U}\right)+u_{0}(X, Y, T)$,

and

$$
\begin{aligned}
l(X, Y, T)=- & \frac{1}{R} \int_{X / U}^{T} p_{Y}(X, Y, \tau) d \tau+\frac{\Omega_{2}}{R} \int_{-\infty}^{X / U} p_{Y}(X=U \tau, Y, \tau) d \tau \\
& +\frac{2 c}{\gamma+1} \frac{M^{2}-1}{M} \int_{-\infty}^{X / U} u_{0 Y}(X=U \tau, Y, \tau) d \tau+v_{0}(X, Y, T),
\end{aligned}
$$

where $u_{0}(X, Y, T)$ and $v_{0}(X, Y, T)$ are related to the prescribed disturbances by Eq. (2.8)

VI. Concluding remarks. Solutions for the diffraction of a plane shock by general two-dimensional weak disturbances are obtained analytically by a method developed for the aerodynamics of blasts. The disturbances which are caused by moving bodies are described by a combination of distributions of sources, doublets and vortices in the two-dimensional case, and by a distribution of point sources in the axisymmetric case. The solution for the disturbance pressure behind an advancing shock in each case is expressed as integrals of known distributions. The shape of diffracted shock and other disturbance quantities are expressed in terms of disturbance pressure and disturbance velocity ahead of the shock.

The present solutions can be applied to many shock diffraction problems of practical interest. The simplest example is the diffraction of a shock by a thin structure, e.g., a wedge with small wedge angle, in still air. Suppose that the shape of the thin structure is given as $Y=h\left(X^{\prime}\right)$ or $h\left[X+\left(U_{0}-U\right) T\right]$; we can determine the corresponding source 
distributions based on this shape. Ahead of the shock, the air is still and there is no disturbance caused by the presence of this structure. The source distribution $f_{0}[X+$ $\left.\left(U_{0}-U\right) T\right]$ for $X>U T$ is zero. Behind the shock, the undisturbed flow velocity is $U_{0}-U$ relative to the structure. The disturbance due to the structure can be measured by the $Y$-component of disturbance velocity

$$
v(X \leq U T, Y \rightarrow 0, T)=\left(U_{0}-U\right) h^{\prime}\left[X+\left(U_{0}-U\right) T\right] .
$$

This disturbance velocity component should be equal to the value due to a distribution of sources on the $X$-axis given by

$$
v(X \leq U T, Y \rightarrow 0, T)=\frac{1}{2} f\left[X+\left(U_{0}-U\right) T\right] .
$$

Equating Eqs. (6.1) and (3.11), we have the corresponding source distribution behind the shock:

$$
f\left[X+\left(U_{0}-U\right) T\right]=2\left(U_{0}-U\right) h^{\prime}\left[X+\left(U_{0}-U\right) T\right] .
$$

If we substitute Eq. (6.2) and $f_{0}=0$ into our general solution in Secs. IV and V, we will have exactly the same result as the two-dimensional solution given in [1].

Using a similar procedure, we can determine an equivalent point source distribution that represents a slender axisymmetric body. The results of diffraction of a shock by this slender axisymmetric body is consistent with those in [1]. For the case of moving bodies, the distributions ahead of a shock is no longer zero. Their strengths can be determined from the relative flow velocity and the body shape. Results for shock diffraction of moving bodies will be presented later. ${ }^{2}$

\section{REFERENCES}

[1] H. F. Ludloff, On aerodynamics of blasts, Advances in Applied Mechanics, vol. 3, Academic Press, New York, 1953, pp. 109-144

[2] G. N. Ward, Linearized theory of steady high-speed flow, Cambridge Univ. Press, London, 1955, p. 21

[3] Y. S. Pan, Solutions for the diffraction of a plane wave by general two-dimensional disturbances, New York University, NYU-ASL-68-42, Oct. 1968

[4] A. E. Puckett, Supersonic wave drag of thin airfoils, J. Aeronaut Sci. 13, 475-484 (1946)

[5] B. B. Baker and E. T. Copson, The mathematical theory of Huygens' principle, Oxford Univ. Press, New York, 1939, p. 36

[6] L. Ting and M. Gunzburger, Diffraction of shock wave by moving thin wing, AIAA Paper 69-647, 1969

Recently, Tine and Gunzburger [6] have extended and applied similar source solutions to the problem of three-dimensional moving wings. 